\title{
CONCOMITANT LAPAROSCOPIC TREATMENT FOR PRIMARY ALDOSTERONISM AND CHOLELITHIASIS
}

\author{
Jun Horiguchi ${ }^{1}$, Yuichi Iino ${ }^{2}$, Toshirou Ikeya ${ }^{3}$, \\ Hiroyuki Takei ${ }^{1}$, Michio Maemura ${ }^{1}$, Hiroshi Matsumoto ${ }^{1}$, \\ Izumi Takeyoshi ${ }^{1}$, Susumu Ohwada ${ }^{1}$, Takao Yokoe $^{2}$, \\ Yasuo Morishita ${ }^{1}$ \\ Second Department of Surgery ${ }^{1}$ and Department of Emergency and Critical Care Medicine ${ }^{2}$, \\ Gunma University School of Medicine, 3-39-15 Showa-machi, Maebashi, Gunma 371-8511, Japan \\ Department of Surgery ${ }^{3}$, Maebashi Red Cross Hospital, 3-21-36 Asahi-cho, Maebashi, Gunma 371, Japan
}

\begin{abstract}
We present the case of a 57-year-old female with cholelithiasis and primary aldosteronism which were successfully treated using concomitant laparoscopic surgery. The operative time was 165 minutes, and intraoperative bleeding was less than $20 \mathrm{ml}$. There was no complication associated with the operation. Concomitant laparoscopic cholecystectomy and adrenalectomy were safely performed in a patient with cholelithiasis and adrenal tumor, and thus this procedure may be the treatment of choice for such a patient.
\end{abstract}

Key words : Laparoscopic surgery, Adrenalectomy, Cholecystectomy

(Kitakanto Med.J. 48 (5) : 359 362, 1998)

\section{INTRODUCTION}

Laparoscopic surgery, particularly cholecystectomy ${ }^{1 \sim 3)}$ has recently become popular in abdominal surgery. Because of the short history of laparoscopic adrenalectomy, the basis for selecting appropriate patients and which adrenal lesions might be appropriate for a laparoscopic approach has not been established. We present a patient with cholelithiasis and a right adrenal tumor which were successfully treated using concomitant laparoscopic cholecystectomy and right adrenalectomy.

\section{CASE REPORT}

A 57-year-old female had received medication for hypertension for 13 years. A medical examination indicated hypopotassemia and the patient was referred to an out-patient clinic of the Gunma University Hospital. Serum aldosterone levels were high and renin activity was low, and an abdominal $\mathrm{CT}$ revealed a mass in the right adrenal gland, cholelithiasis and hepatic cysts. An MRI also revealed a mass in the right

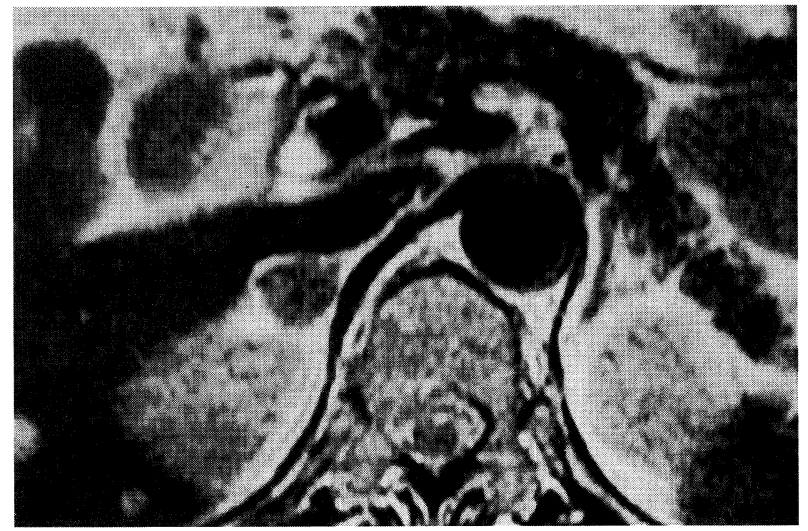

Fig. 1 MRI findings: A mass in the right adrenal gland

adrenal gland (Fig. 1). An adosterol scintigram showed an increased accumulation at the right adrenal gland (Fig. 2). Venous sampling examination indicated an elevated level of serum aldosterone at the right adrenal vein. During hospitalization, the patient complained of right hypochondralgia. Ultrasonography demonstrated a strong internal echo and wall thickness of the gallbladder (Fig. 3). The pain due to cholelith- 


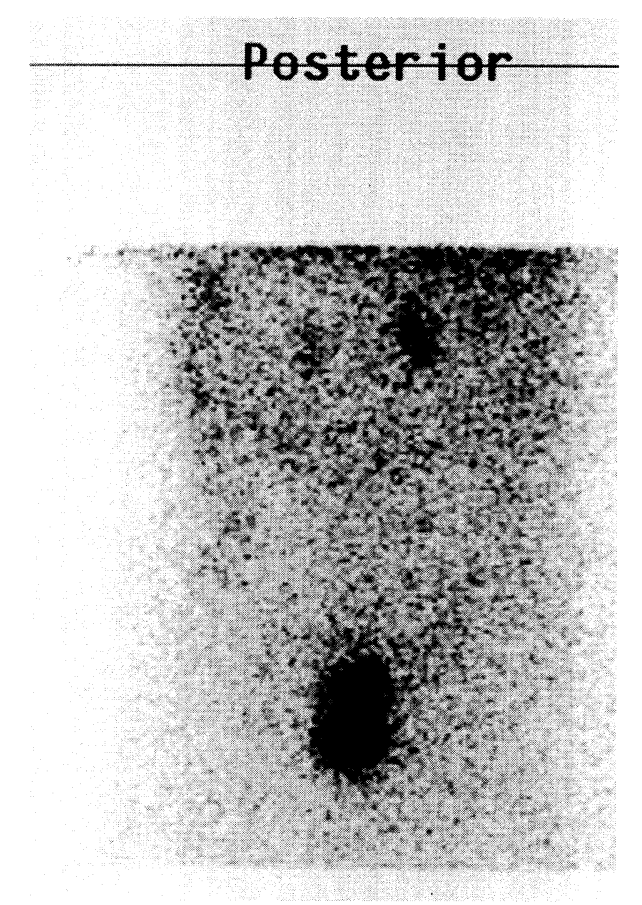

Fig. 2 Adosterol scintigram showing an increased accumulation at the right adrenal gland

iasis disappeared with conservative therapy. Endoscopic retrograde chalangiopancreatography revealed a gallbladder stone without any abnormality in the common bile duct. The patient was diagnosed with primary aldosteronism due to a right adrenal tumor and cholecystitis with a gallbladder stone, and a right adrenalectomy and cholecystectomy were planned for May, 1997.

Under general anesthesia, a small infraumbilical incision was made. A needle was placed into the abdominal cavity for $\mathrm{CO}_{2}$ insufflation. A video laparoscope was introduced into the abdominal cavity through the subumbilical trochar. An 11-mm trochar was placed in a subxiphoid, midline position. In addition, a 12-mm trochar was placed in the right anterior axillary line near the umbilical level, and a 5 -mm trochar placed in the right subcostal anterior axillary line. Standing at a patient's left side, a surgeon maintained grasping forceps placed in the right subcostal anterior axillary line and dissecting forceps in the subxiphoid midline. The cholecystectomy was performed first. As the inflammation of the gallbladder was mild, the gallbladder was easily removed in a retrograde fashion. Following cholecystectomy, the patient was placed in the left lateral decubitus position. An $12-\mathrm{mm}$ trochar was placed in the right midclavicular line near the umbilical level. The liver was retracted through the trochar in the right anterior axillary line near the umbilical level, and the colon was retracted through the trochar in the right midclavicular line near

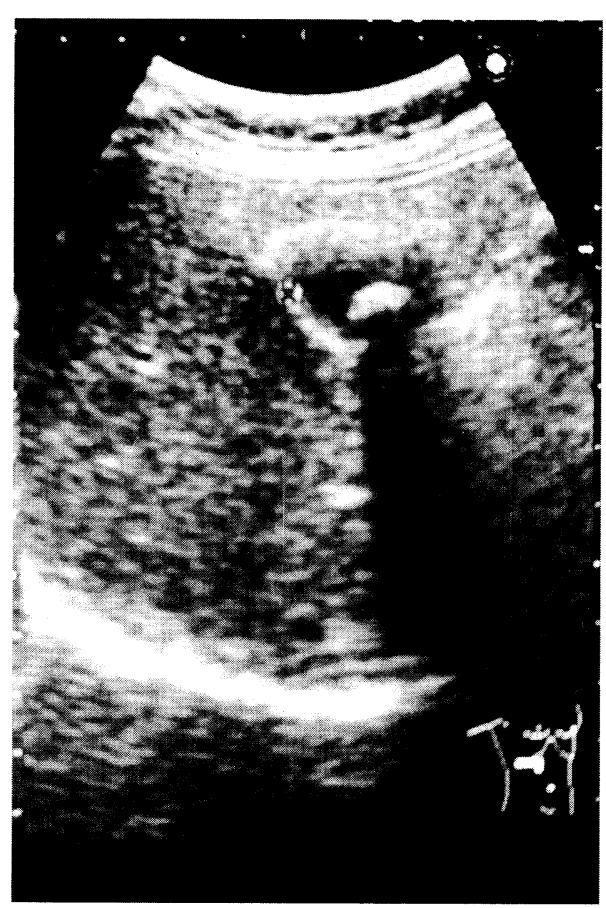

Fig. 3 US findings showing a strong internal echo and the wall thickness of the gallbladder

the umbilical level. The vena cava was exposed, and the renal vein was identified as a landmark. The right adrenal vein from the vena cava was visible and divided with two clips. Arterial branches of the inferior phrenic artery were coagulated and divided using electrocautery. The right adrenal gland was dissected, and removed. A drainage tube was introduced from the port in the right subcostal anterior axillary line (Fig.4). The operative time was 165 minutes, and intraoperative bleeding was less than $20 \mathrm{ml}$.

The gallbladder included a $1.5 \mathrm{~cm}$ stone (Fig.5). The adrenal tumor, $1.7 \mathrm{~cm}$ in size, was goldenyellowish and solid (Fig.6). There were no complications associated with the operation. The patient was

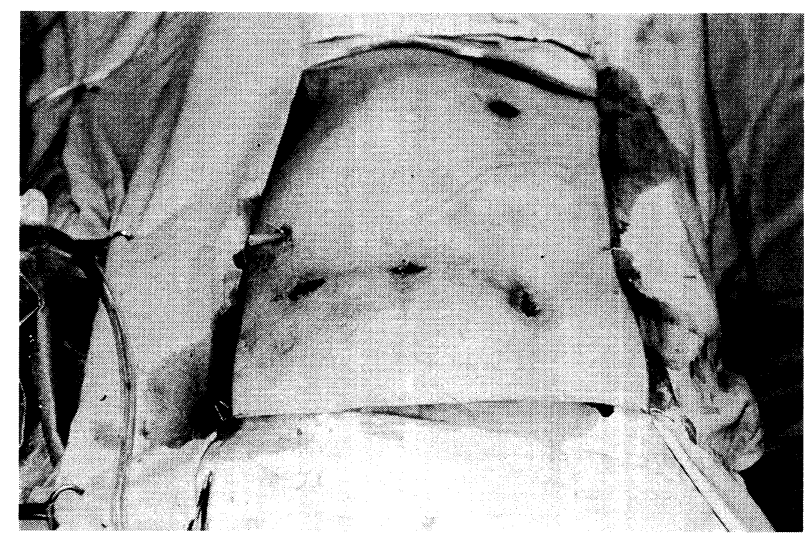

Fig. 4 A drainage tube was introduced from the port in the right subcostal anterior axillary line, and skin incisions are shown. 


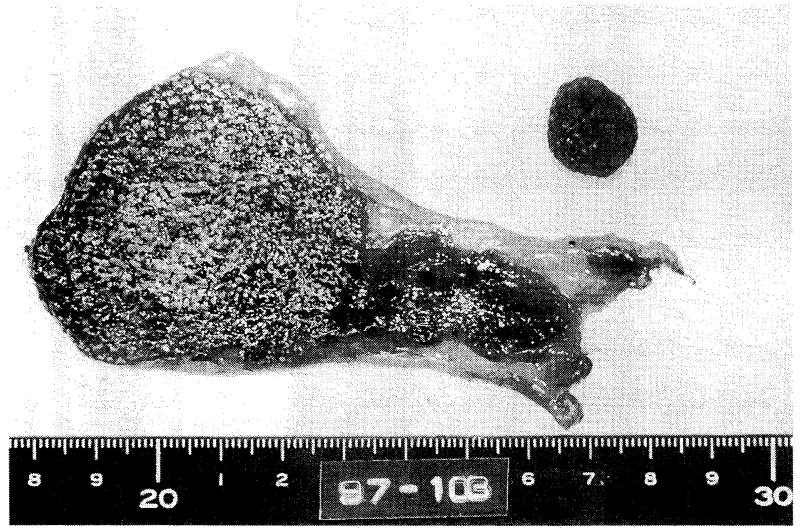

Fig. 5 The wall of the gall bladder changed with cholesterosis and a $1.5 \mathrm{~cm}$ stone in the gallbladder.

ambulatory on the following day and was discharged on the sixth day after the operation. The histological diagnosis of the adrenal tumor was adrenal cortical adenoma. Chronic cholecystitis and cholesterol polyps with cholelithiasis had formed in the gallbladder. There were no malignant findings.

\section{DISCUSSION}

Advances in endoscopic equipment have encouraged the development of endoscopic surgical treatment for abdominal surgery. Laparoscopic cholecystectomy was first introduced into the practice of biliary surgery in South America ${ }^{1)}$ and in France ${ }^{2)}$. The large size of the prospective analysis of the Southern Surgeons Club $^{3)}$ showed that results of laparoscopic cholecystectomy could be favorably compared with those of conventional cholecystectomy with respect to mortality, complications, and length of hospital stay. Recently laparoscopic cholecystectomy has gained widespread acceptance as a safe and effective treatment of cholelithiasis. Laparoscopic adrenalectomy was first reported by Gagner et al. ${ }^{4)}$ in 1992, and it has become the procedure of choice for most benign, functioning and nonfunctioning adrenal masses. As a result of the comparison study between an open transperitoneal and a laparoscopic operation, the laparoscopic adrenalectomy has become an acceptable treatment ${ }^{5 \sim 8)}$.

Traditional open cholecystectomy or adrenalectomy usually requires large incisions that cause considerable pain and morbidity. Both cholelithiasis and adrenal tumors are ideal candidates for laparoscopic removal because they are usually benign and no reconstruction is needed. In our patient, there was no abnormality in the common bile duct and inflammatory changes of the gallbladder were mild. The adrenal tumor size was small. Thus concomitant cholecystectomy and adrenalectomy were successfully perfor-

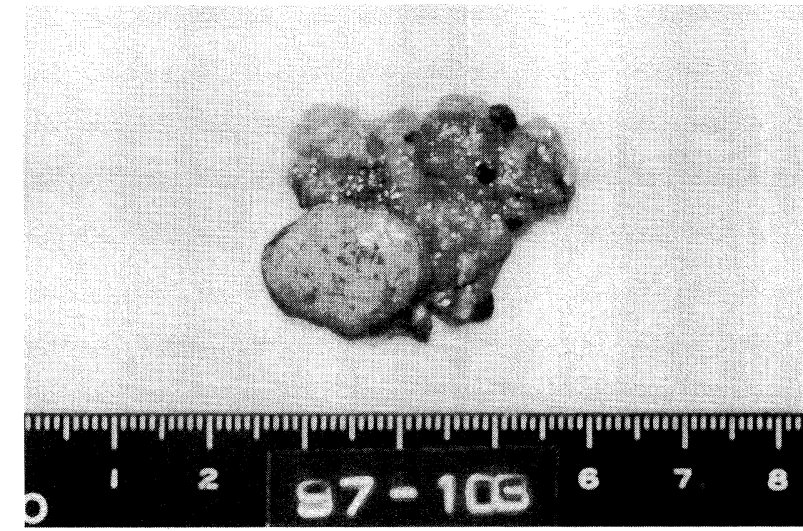

Fig. 6 The adrenal tumor, $1.7 \mathrm{~cm}$ in size, was golden-yellowish and solid.

med using a laparoscopic approach.

Controversy remains as to which approach, either transabdominal or retroperitoneal, is better in a laparoscopic adrenalectomy. Takeda et al. ${ }^{9)}$ reported that retroperitoneal laparoscopic adrenalectomy was feasible for primary aldosteronism. Mercan et al. ${ }^{10)}$ reported that endoscopic retroperitoneal adrenalectomy was a new and safe method of adrenalectomy with less postoperative pain and discomfort, and a shorter postoperative hospitalization and recovery period. Fernandez et al. ${ }^{11)}$ also reported that the retroperitoneal approach might be a better option in patients with previous abdominal surgery and in patients with preexisting cardiorespiratory diseases. We chose a transperitoneal approach because an endoscopic cholecystectomy was concomitantly performed.

The main complication of transperitoneal adrenalectomy is pneumothorax due to injury of the diaphragm, especially in a left adrenalectomy. Naito et a1. ${ }^{7)}$ also reported that the chief complication in a laparoscopic adrenalectomy was pneumothorax secondary to injury of the diaphragm and pleura during dissection of the left adrenal gland using electrocautery.

The mean operative time of laparoscopic adrenalectomy ${ }^{12 \sim 14)}$ has been reported to range from 120 to 572 minutes. Some researchers ${ }^{5,15,16)}$ reported that laparoscopic adrenalectomy took more time compared with an open adrenalectomy, however, the operative time of laparoscopic adrenalectomy may be reduced with experience ${ }^{13)}$. In our case 165 minutes elapsed during both the cholecystectomy and adrenalectomy and the patient was discharged on the sixth day after surgery. Both laparoscopic adrenalectomy and cholecystectomy were safely and effectively performed, and thus this procedure may be the treatment of choice for such a patient. 


\section{REFERENCES}

1) Reddick EJ, Olsen DO. Laparoscopic laser cholecystectomy: a comparison with mini-lap cholecystectomy. Surg Endosc 1989; 3: 131-133.

2) DuBois F, Icard P, Berthelot G, Levard $H$. Coelioscopic cholecystectomy : preliminary report of 36 cases. Ann Surg 1990; 211 : 60-62.

3) The Southern Surgeons Club. A prospective analysis of 1518 laparoscopic cholecystectomies. New Engl J Med 1991; 324: 1073-1078.

4) Gagner M, Lacroix A, Bolte E. Laparoscopic adrenalectomy in Cushing's syndrome and pheochromocytoma. N Engl J Med 1992; 327 : 1033.

5) MacGillivray DC, Shichman SJ, Ferrer FA, Malchoff CD. A comparison of open vs laparoscopic adrenalectomy. Surg Endosc 1996; 10 : 987-990.

6) Brunt LM, Doherty GM, Norton JA, Soper NJ, Quasebarth MA, Moley JF. Laparoscopic adrenalectomy compared to open adrenalectomy for benign adrenal neoplasma. J Am Coll Surg $1996 ; 183: 1-10$.

7) Naito S, Uozumi J, Shimura H, Ichimiya $H$, Tanaka M, Kumazawa J. Laparoscopic adrenalectomy: review of 14 cases and comparison with open adrenalectomy. J Endourol 1995; 9 : 491-495.

8) Prinz RA. A comparison of laparoscopic and open adrenalectomies. Arch Surg 1995; 130: 489-492.

9) Takeda M, Go H, Watanabe R, Kurumada S, Obara K, Takahashi E, Komeyama T, Imai T, Takahashi K. Retroperitoneal laparoscopic adrenalectomy for functioning adrenal tumors: comparison with conventional transperitoneal laparoscopic adrenalectomy. J Urol 1997; 157, 19-23.

10) Mercan S, Seven R, Ozarmagan S, Tezelman S. Endoscopic retroperitoneal adrenalectomy. Surgery $1995 ; 118$ : 1071-1075.

11) Fernandez CL, Saenz A, Benarroch G, Astudillo E, Taura P, Sabater L. Laparoscopic unilateral and bilateral adrenalectomy for Cushing's syndrome. Transperitoneal and retroperitoneal approaches. Ann Surg 1996; 224 : 727-734.

12) Takeda M, Go H, Imai $T$, Nishiyama $T$, Morishita H. Laparoscopic adrenalectomy for primary aldosteronism : report of initial ten cases. Surgery 1994 ; $115:$ 621-625.

13) Walmsley D, McIntyre R, Sawers HA, Shaw JA, Webster J, Krukowski ZH, Bevan JS. Laparoscopic trans-peritoneal adrenalectomy: a preliminary of 14 adrenalectomies. Clin Endocrinol Oxf 1996 ; 45 : 141-145.

14) Marescaux J, Mutter D, Wheeler MH. Laparoscopic right and left adrenalectomies. Surgical procedures. Surg Endosc 1996; 10 : 912-915.

15) Naito $S$, Uozumi J, Ichimiya $H$, Tanaka $M$, Kimoto K, Takahashi K, Ohta J, Tanaka M, Kumazawa J. Laparoscopic adrenalectomy: comparison with open adrenalectomy. Eur Urol 1994 ; 26 : 253-257.

16) Thompson GB, Grant CS, van Heerden JA, Schlinkert RT, Young Jr WF, Farley DR, Ilstrup DM. Laparoscopic versus open posterior adrenalectomy: A case-control study of 100 patients. Surgery $1997 ; 122$ : 1132-1136. 